\title{
Rarefied Gas Flow Through an Orifice at Finite Pressure Ratio
}

\author{
Felix Sharipov \\ Departamento de Física, Universidade Federal do Paraná \\ Caixa Postal 19044, 81531-990 Curitiba, Brazil \\ Email: sharipov@fisica.ufpr.br; Website: fisica.ufpr.br/sharipov/
}

\begin{abstract}
The rarefied gas flow through a thin orifice is studied on the basis of the direct simulation Monte Carlo method. The mass flow rate and the flowfield have been calculated over the whole range of the Knudsen number for various values of the pressure ratio. It has been found that at any value of the pressure ratio a significant variation of the flow rate occurs in the transition regime. In the hydrodynamic regime the flow rate tends to a constant value. In the case of finite pressure ratio the flowfield qualitatively differs from that of the outflow into vacuum, namely, vortices appear in the down flow container by approaching to the hydrodynamic regime. Then, in the deep hydrodynamic regime the gas flow forms a strong jet. A comparison of the numerical results with experimental data available in the open literature has been performed.
\end{abstract}

\section{INTRODUCTION}

The rarefied gas flow through a thin orifice has a great practical interest. This kind of the flow is realized in vacuum equipment, microfluidics, spacecraft designing and in many other applications. As it was noted in the review [1], in spite of the great practical importance, till now no reliable results on the orifice flow are available in the open literature for the wide ranges of the gas rarefaction and of the pressure ratio.

The statement the problem is very simple. Consider an orifice in an infinitesimally thin partition that separates two semi-infinite containers. One of them contains a monatomic gas at a pressure $P_{0}$, while the other container is maintained at a smaller pressure $P_{1}$, i.e. $P_{1}<P_{0}$. The temperatures of the gas in both containers are equal to $T_{0}$. We are going to calculate the mass flow rate $\dot{M}$ through the orifice and the flowfield in both containers.

The solution of the problem is determined by two main parameters: the pressure ratio $P_{1} / P_{0}$ and the gas rarefaction $\delta$ defined as

$$
\delta=\frac{a P_{0}}{\mu_{0} v_{0}}, \quad v_{0}=\left(\frac{2 k T_{0}}{m}\right)^{1 / 2},
$$

where $a$ is the orifice radius, $\mu_{0}$ is the stress viscosity at the temperature $T_{0}, v_{0}$ is the most probable molecular velocity at the same temperature, $m$ is the molecular mass of the gas, $k$ is the Boltzmann constant. So, the limit $\delta=0$ corresponds to the free molecular regime and the opposite limit, i.e. $\delta \rightarrow \infty$, describes the hydrodynamic regime.

In the previous papers [2, 3], a gas outflow into vacuum, i.e. the case of $P_{1} / P_{0}=0$, was considered. The aim of the present work is to carry out a direct numerical simulation of the rarefied gas flow through a thin orifice over the whole range of the rarefaction parameter $\delta$ for finite values of the pressure ratio $P_{1} / P_{0}$. The numerical results will be given in terms of the reduced flow rate defined as

$$
W=\frac{\dot{M}}{\dot{M}_{0}}, \quad \dot{M}_{0}=\sqrt{\pi} a^{2} \frac{P_{0}}{v_{0}},
$$

where $\dot{M}$ is the mass flow rate at any gas rarefaction and at any pressure ratio $P_{1} / P_{0}, \dot{M}_{0}$ is the mass flow rate in the free molecular regime $(\delta=0)$ in the case of outflow into vacuum, i.e. at $P_{1} / P_{0}=0$. 


\section{METHOD OF SOLUTION}

Like the previous papers [2, 3], the problem in question was solved by the Direct Simulation Monte Carlo (DSMC) method [4]. The hard sphere molecular model and the diffuse gas-surface interaction were assumed. To simulate the intermolecular collisions, the so-called NTC method (see Ref. [4], p. 219) was used. In every time step the number of particles passed through the orifice from the upflow container to the downflow one $N^{+}$and the number of particles passed through the orifice in the opposite direction $N^{-}$were counted. Then, the flow rate was calculated from the number of particles passed through the orifice in both directions, i.e. via the quantity $\left(N^{+}-N^{-}\right)$. To guarantee the statistical scattering of the flow rate would be less than $1 \%$ every calculation continued until the condition

$$
\frac{\sqrt{N^{+}}+\sqrt{N^{-}}}{N^{+}-N^{-}}<0.001
$$

was satisfied.

The number of model particles fluctuated during the calculation, but it was maintained about $10^{6}$. The other parameters of the numerical scheme, i.e. the cell size and the time increment were chosen so that to provide the numerical error of the flow rate less than $1 \%$. To estimate the numerical error, test calculations were carried out for four values of the rarefaction parameter $\delta: 0.1,1,10$ and 100. In these calculations the number of model particles was increased up to $5 \times 10^{6}$, the cell size was reduces as twice, and the time increment was reduced as five times.

It should be note, that the CPU time drastically increases by increasing the rarefaction parameter $\delta$ because the larger value of $\delta$ leads to a larger number of intermolecular collisions and requires the use of denser numerical grid. For a fixed value of $\delta$ the $\mathrm{CPU}$ time increases by increasing the pressure ratio $P_{1} / P_{0}$. If the pressure ratio $P_{1} / P_{0}$ is close to unity the number of samples necessary to satisfy the condition (3) increases. So, the case of $\delta=200$ and $P_{1} / P_{0}=0.9$ requires the longest $\mathrm{CPU}$ time among all cases considered here.

\section{RESULTS AND DISCUSSION}

The calculations were carried out for four values of the pressure ratio $P_{1} / P_{0}: 0,0.1,0.5$, and 0.9 . The first value corresponds to the outflow into vacuum. Partially, such results were presented in the previous papers $[2,3]$. So, this case is characterized as a strong non-equilibrium flow. The second value of $P_{1} / P_{2}$ also corresponds to a strong nonequilibrium flow, but this case allows us to study the influence of a presence of small quantity of the gas in the downflow container. The third value of the pressure ratio represents a transition between the strong non-equilibrium flow and a weak one. Finally, the gas flow at the fourth value of $P_{1} / P_{0}$ can be characterized as weakly non-equilibrium one. In this case the relative pressure drop $\left(P_{0}-P_{1}\right) / P_{0}$ is equal to 0.1 .

The numerical results on the reduced flow rate $W$ are presented in Fig. 1 by the non-filled symbols. In the free molecular regime $(\delta=0)$, the numerical value of $W$ tends to its theoretical value given as $W=\left(1-P_{1} / P_{0}\right)$. In the hydrodynamic regime, the flow rate $W$ also tends to a constant value, which depends on the pressure ratio $P_{1} / P_{0}$. The significant variation of the flow rate occurs in the interval of the rarefaction parameter $\delta$ from 0.5 to 20 for any pressure ratio $P_{1} / P_{0}$. The relative variation of the mass flow rate increases by increasing the pressure ratio. In the case of outflow into vacuum $\left(P_{1} / P_{0}=0\right)$ the value of the flow rate $W$ in the hydrodynamic regime $(\delta \rightarrow \infty)$ is larger as 1.5 times than its value in the free molecular regime $(\delta=0)$, while for the small pressure difference $P_{1} / P_{0}=0.9$ the ratio between the flow rates in these two limits is about 6.6.

The experimental data for two values of the pressure ratio $P_{1} / P_{1}=0.1$ and 0.5 reported by Sreekanth [5] are shown in Fig. 1 by the filled symbol. The numerical results are in a fine agreement with the experimental data. It should be noted that in the open literature there is a lack of reliable experimental data on the orifice flow for wide ranges of the gas rarefaction and pressure ratio.

The density distributions $n / n_{0}$ for four values of the rarefaction parameter $\delta$ are presented in Figs. 2, 3, 4, and 5 for $P_{1} / P_{0}=0,0.1,0.5$, and 0.9 , respectively. It can be proved that in the free molecular regime the density distribution is symmetric, i.e.

$$
n(x, y, z)=n_{0}+n_{1}-n(-x, y, z),
$$

where $n_{0}=P_{0} / k T_{0}$ and $n_{1}=P_{1} / k T_{0}$. Here, the Cartesian coordinates have been introduced so as the partition is placed at $x=0$. From Figs. 2-5 one can see that near the free molecular regime $(\delta=0.1)$ and in the transition regime $(\delta=1)$ the density distributions are very close to that described by Eq.(4). In the hydrodynamic regime the distributions are 


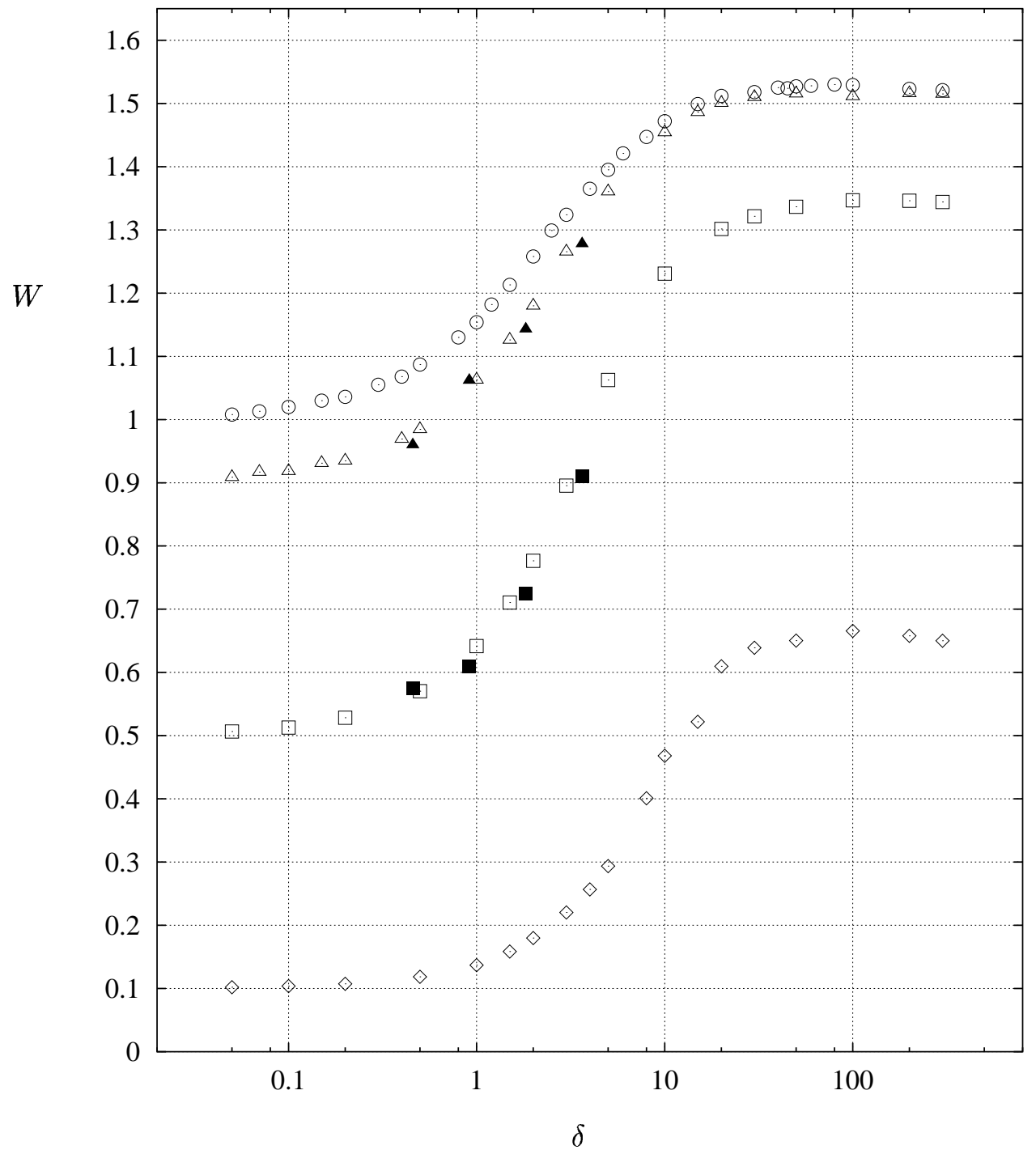

FIGURE 1. Reduced flow rate $W$ vs rarefaction parameter $\delta$ : non-filled symbols - present results; filled symbols - experimental data [5]; circles $-P_{1} / P_{0}=0$; triangles $-P_{1} / P_{0}=0.1$; squares $-P_{1} / P_{0}=0.5$; diamonds $-P_{1} / P_{0}=0.9$ 

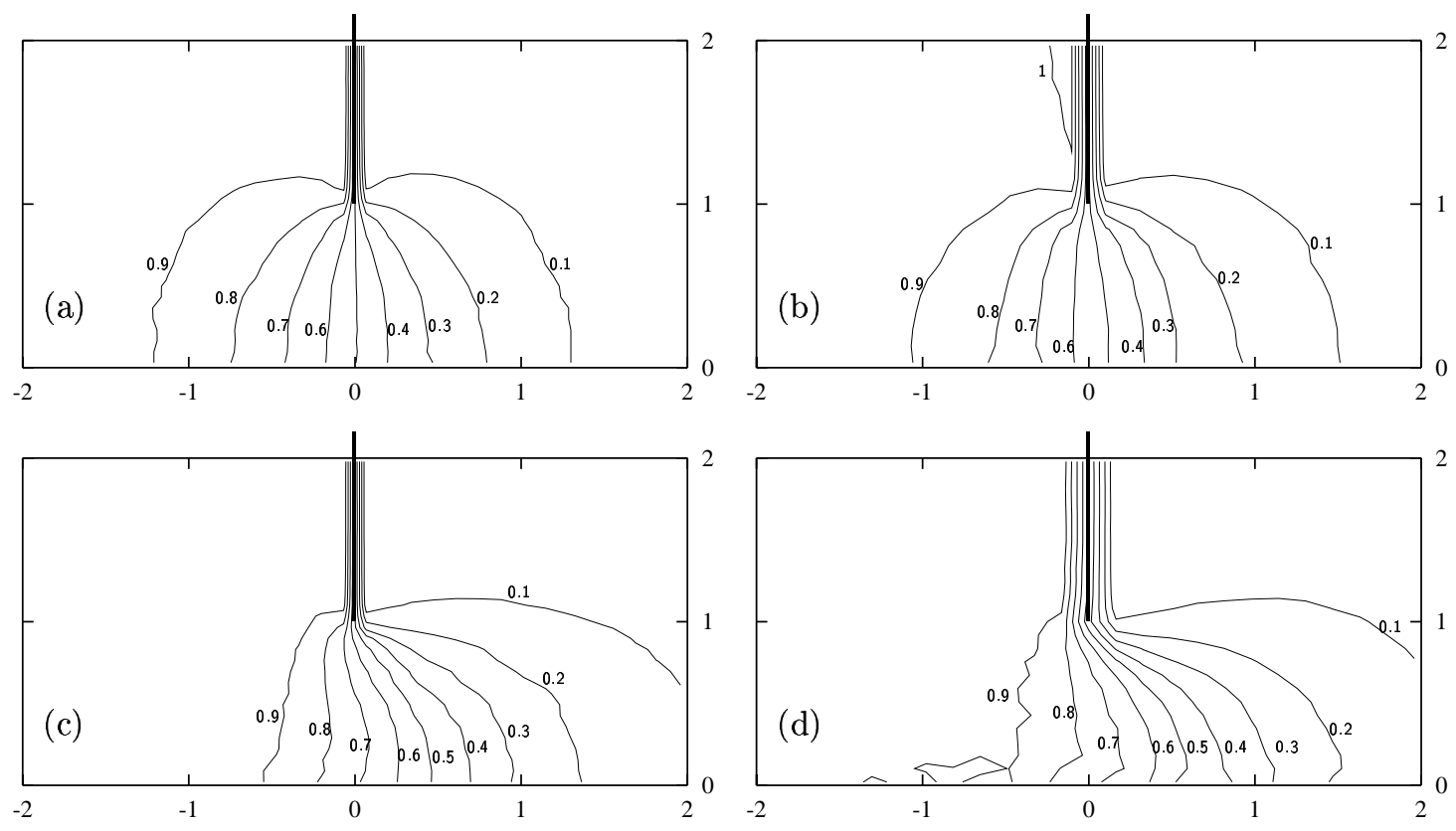

FIGURE 2. Density distribution $n / n_{0}$ at $P_{1} / P_{0}=0$ : (a) $-\delta=0.1$; (b) $-\delta=1$; (c) $-\delta=10$; (d) $-\delta=100$
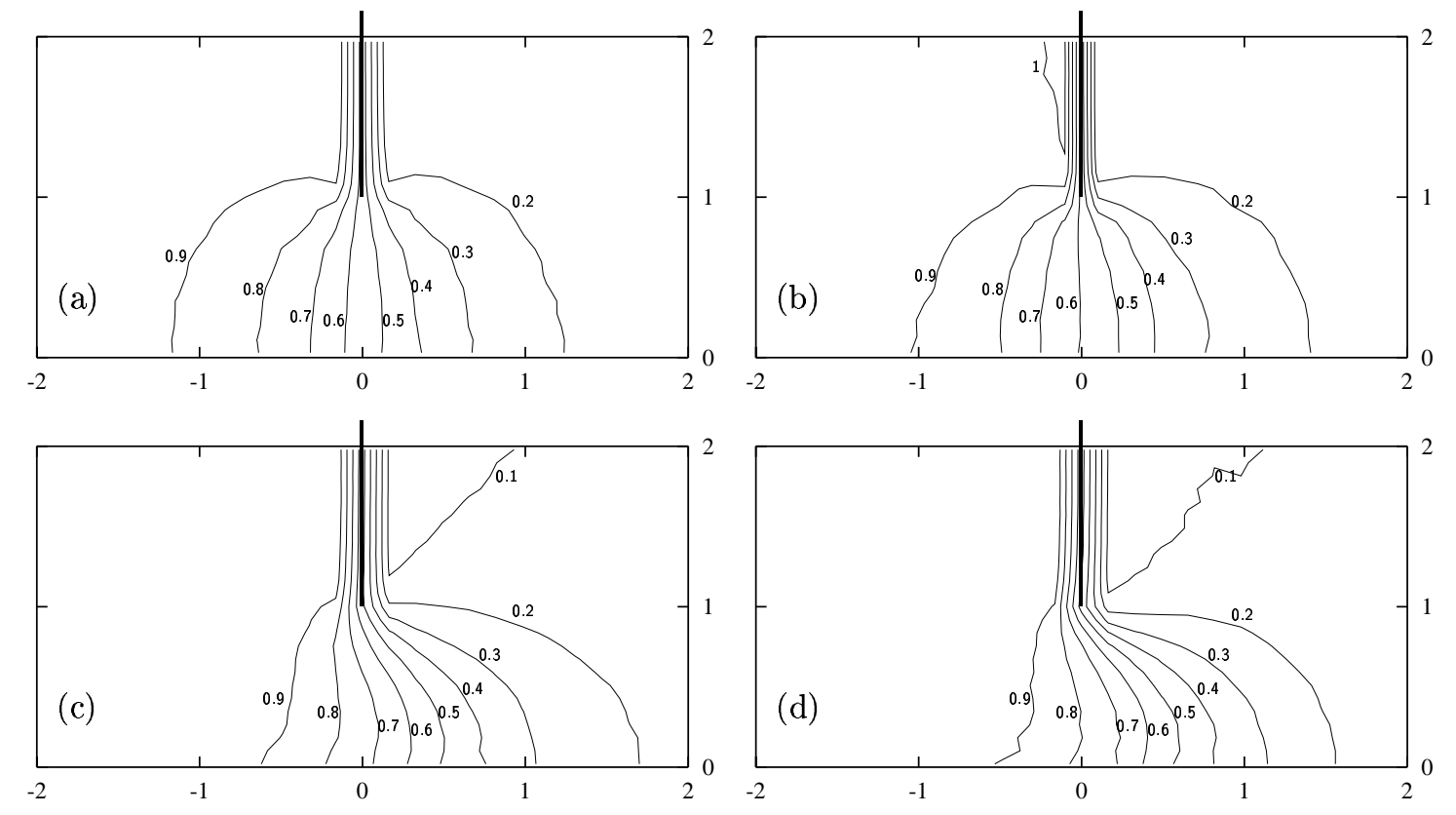

FIGURE 3. Density distribution $n / n_{0}$ at $P_{1} / P_{0}=0.1$ : (a) $-\delta=0.1$; (b) $-\delta=1$; (c) $-\delta=10$; (d) $-\delta=100$ 

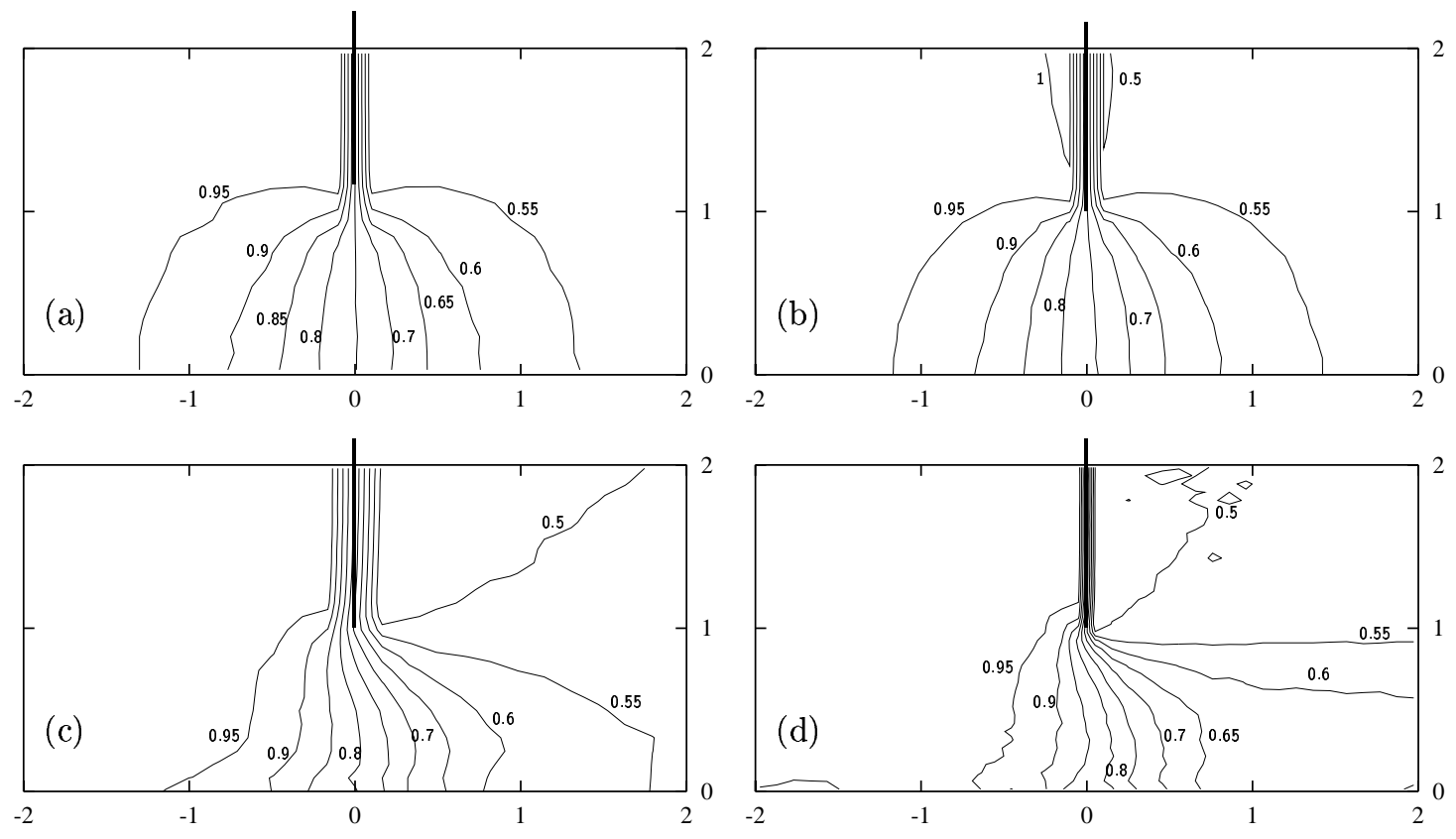

FIGURE 4. Density distribution $n / n_{0}$ at $P_{1} / P_{0}=0.5$ : (a) $-\delta=0.1$; (b) $-\delta=1$; (c) $-\delta=10$; (d) $-\delta=100$
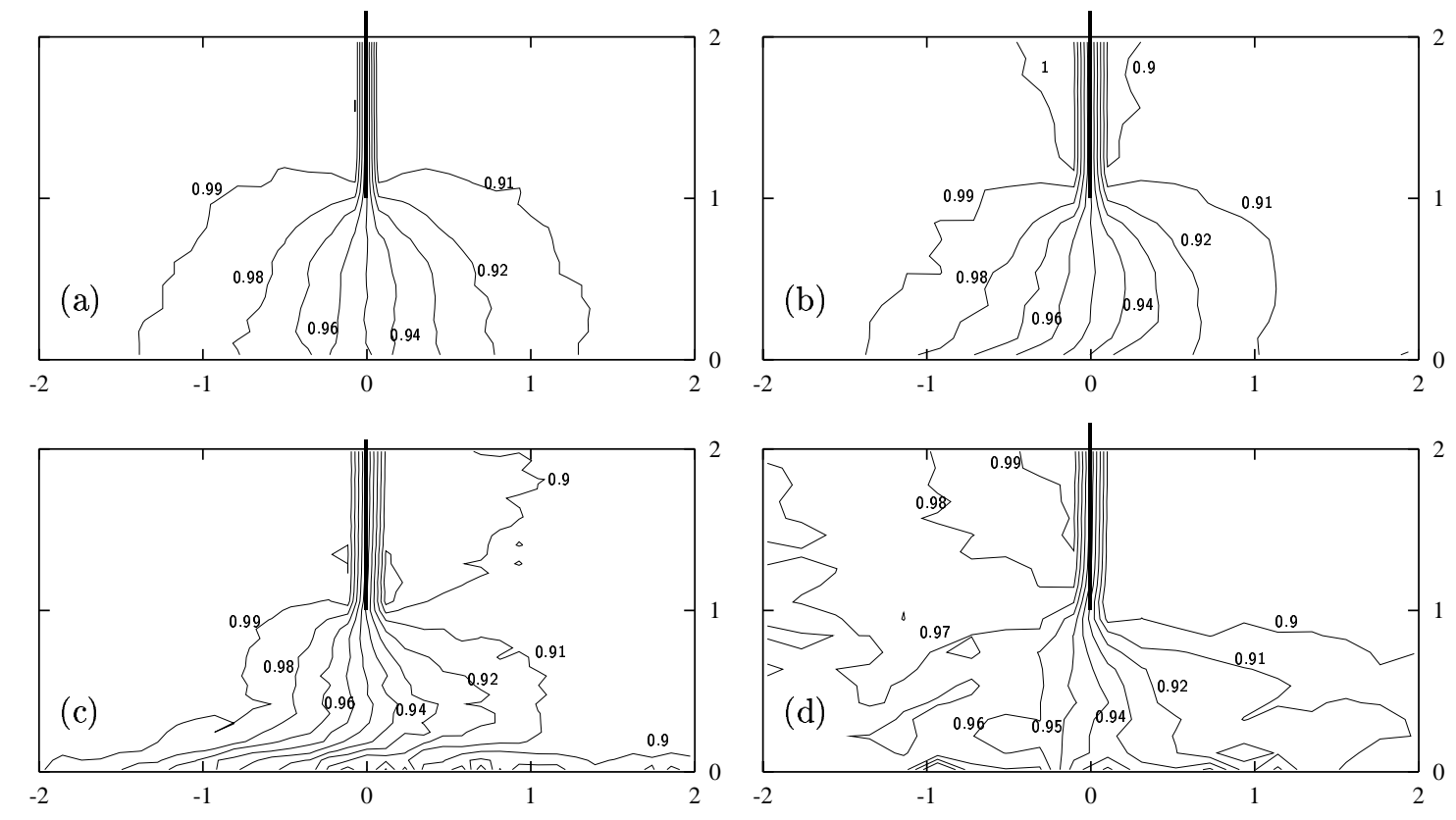

FIGURE 5. Density distribution $n / n_{0}$ at $P_{1} / P_{0}=0.9$ : (a) $-\delta=0.1$; (b) $-\delta=1$; (c) $-\delta=10$; (d) $-\delta=100$ 

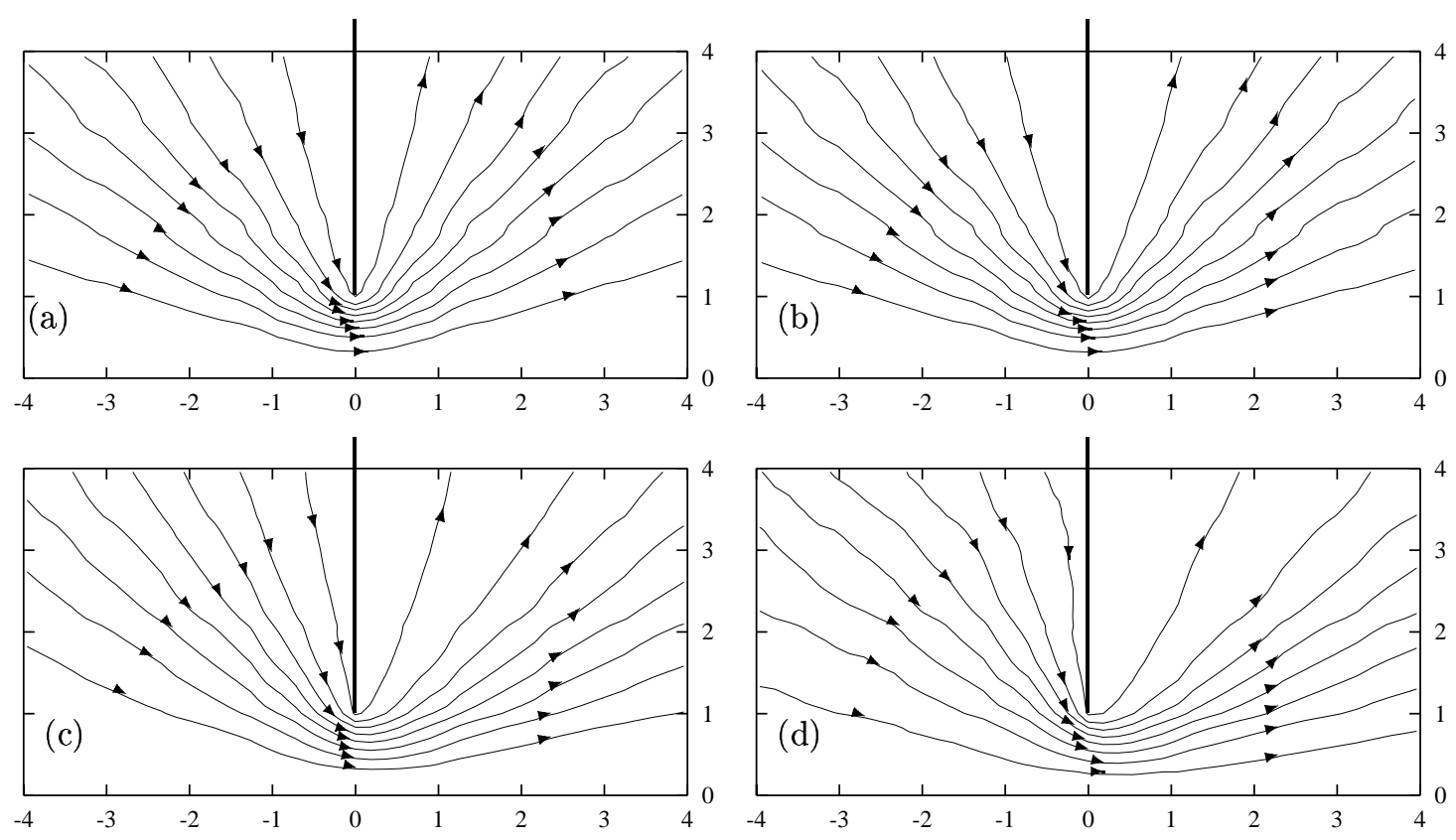

FIGURE 6. Streamlines at $P_{1} / P_{0}=0$ : (a) $-\delta=0.1$; (b) - $\delta=1$; (c) - $\delta=10$; (d) - $\delta=100$
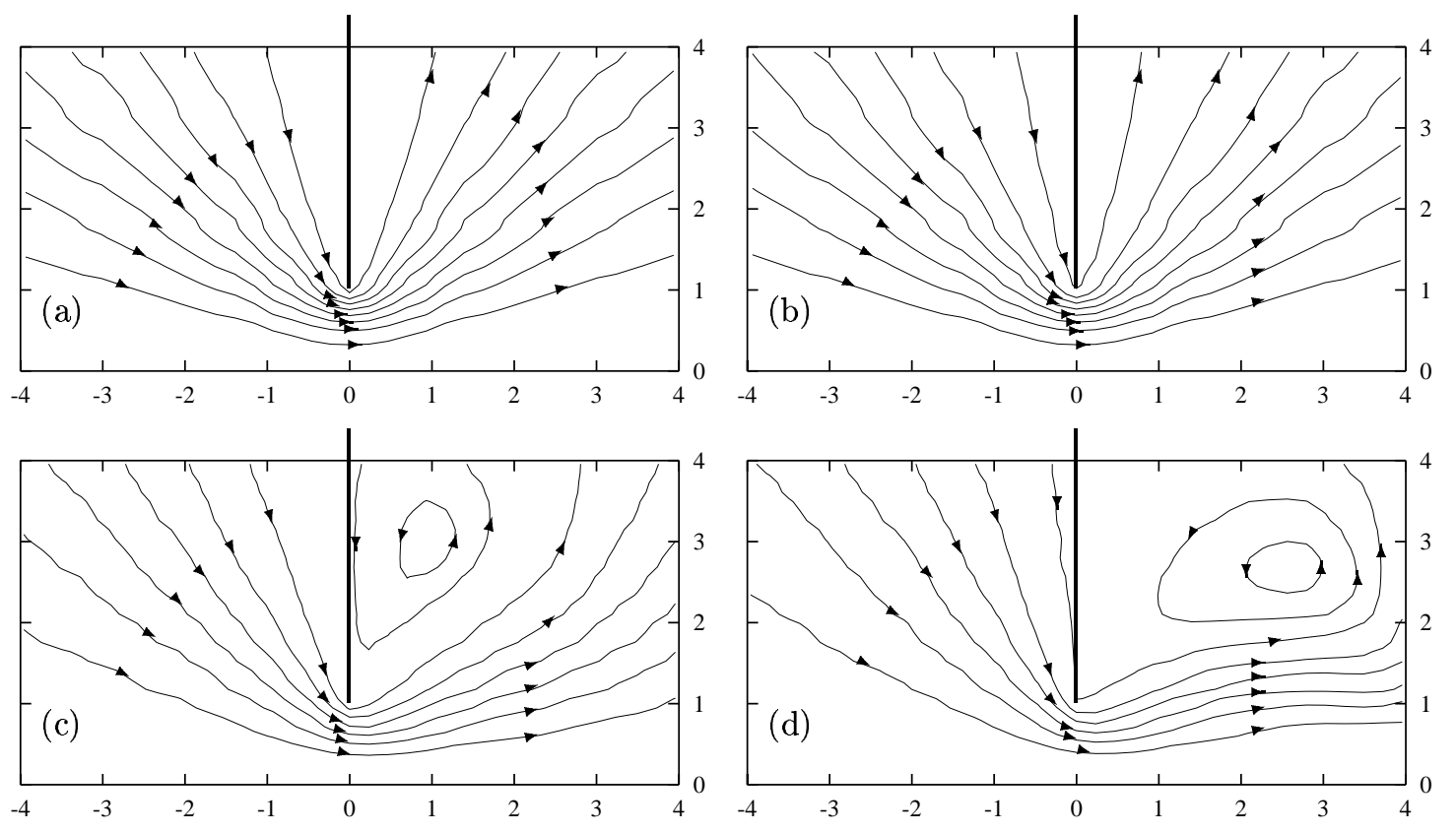

FIGURE 7. Streamlines at $P_{1} / P_{0}=0.1$ : (a) $-\delta=0.1$; (b) $-\delta=1$; (c) $-\delta=10$; (d) $-\delta=100$ 

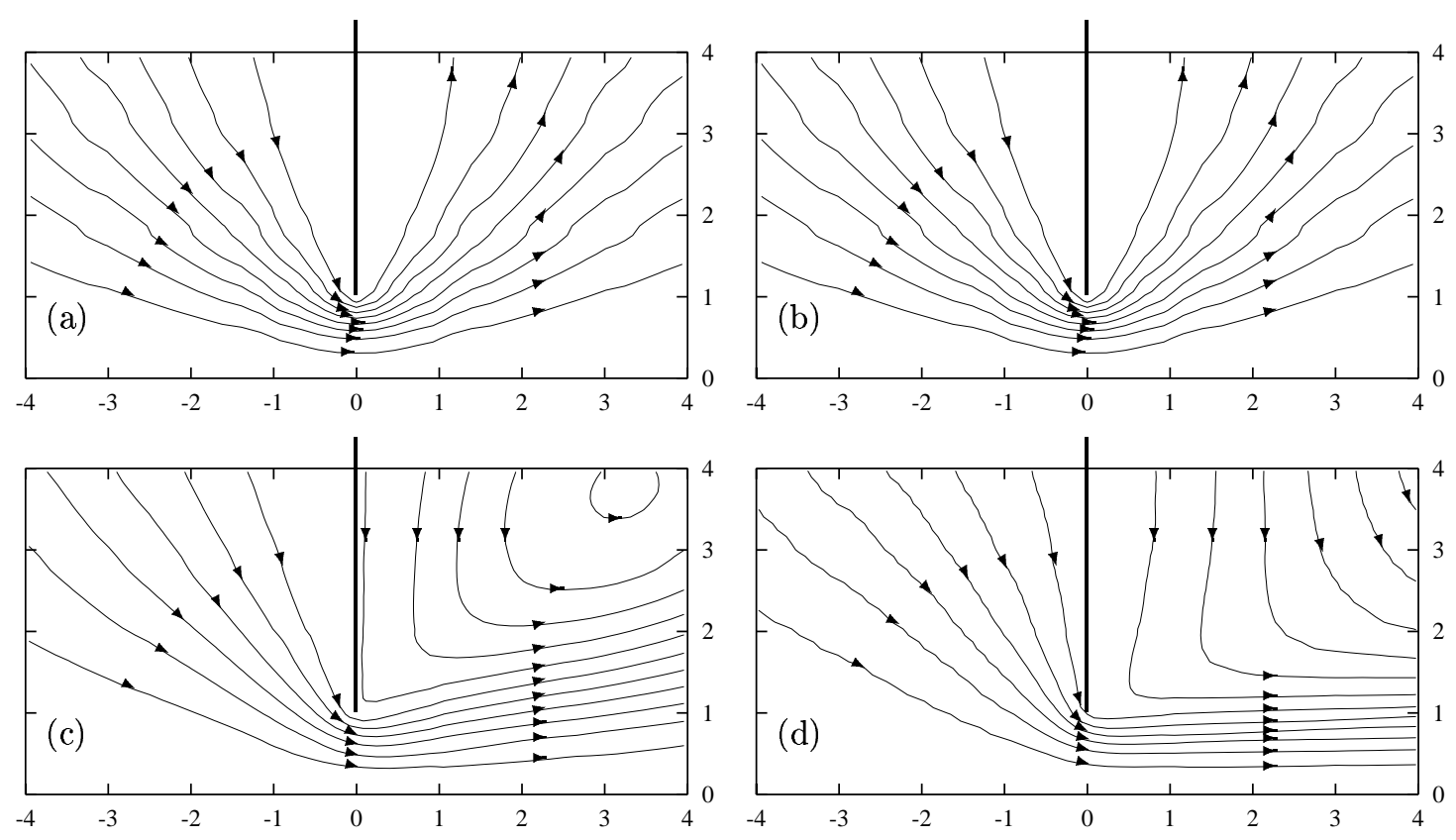

FIGURE 8. Streamlines at $P_{1} / P_{0}=0.5$ : (a) $-\delta=0.1$; (b) $-\delta=1$; (c) $-\delta=10$; (d) $-\delta=100$
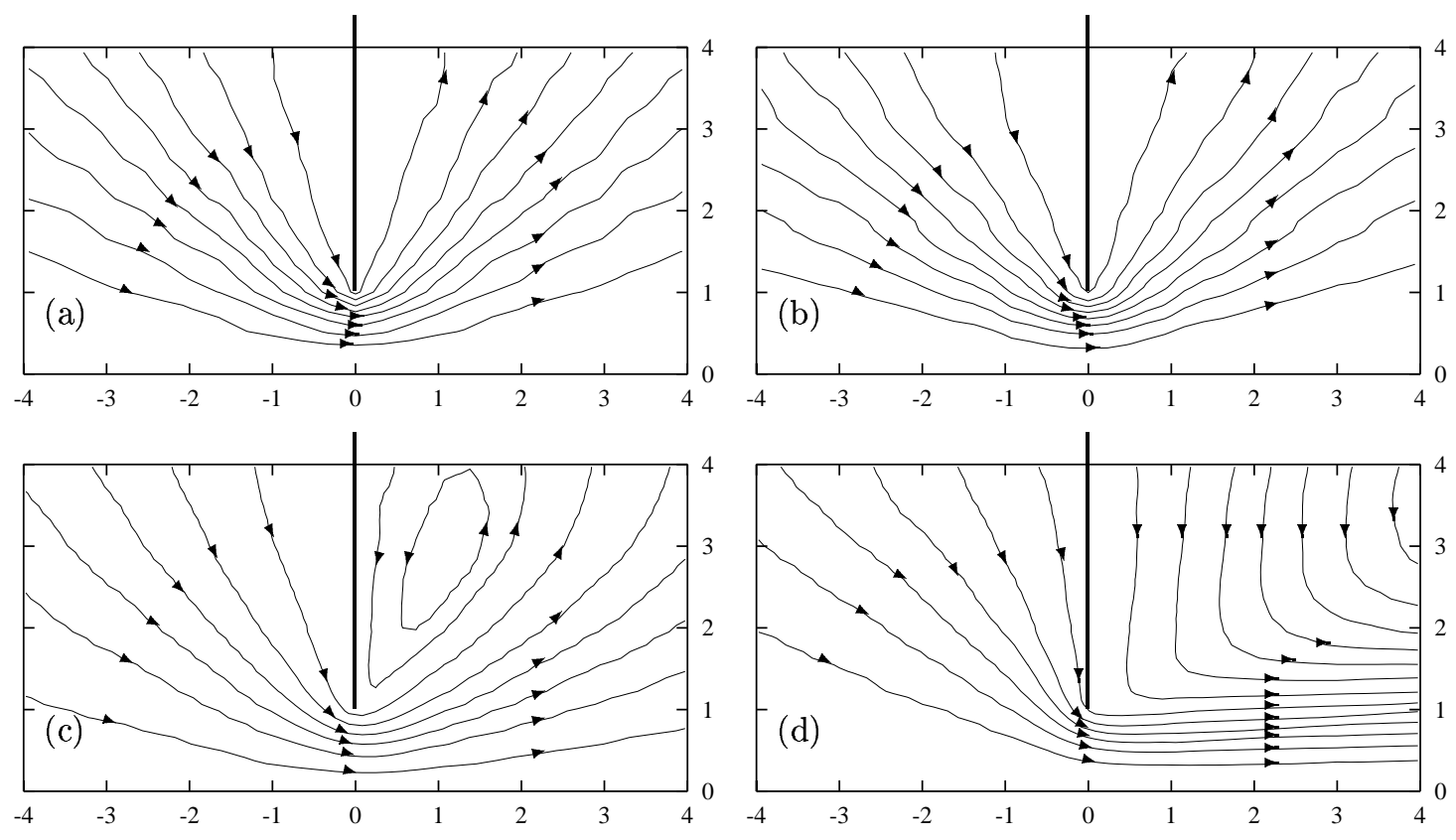

FIGURE 9. Streamlines at $P_{1} / P_{0}=0.9$ : (a) $-\delta=0.1$; (b) $-\delta=1$; (c) $-\delta=10$; (d) $-\delta=100$ 
quite asymmetric. Also we note that the fluctuation of the density related to the statistical scattering becomes significant at the small pressure drop, i.e. at $P_{1} / P_{0}=0.9$, even if the number of samples is very large.

The streamlines for the same values of the rarefaction parameter $\delta$ are given in Figs. 6, 7, 8, and 9 at $P_{1} / P_{0}=0$, $0.1,0.5$, and 0.9 , respectively. Near the free molecular regime $(\delta=0.1)$ and in the transition regime $(\delta=1)$ the streamlines are symmetric. However, in the hydrodynamic regime $(\delta=10$ and 100$)$ the flow begins to form vortices in the downflow container if the pressure ratio is finite, i.e. at $P_{1} / P_{0} \geq 0.1$, while in the case of outflow into vacuum $\left(P_{1} / P_{0}=0\right)$ no vortices appear even at the large value of the rarefaction parameter, i.e. at $\delta=100$. Note, that at $P_{1} / P_{0}=0.1$ the flow rate $W$ corresponding to the hydrodynamic regime $(\delta=100)$ is practically the same as that for $P_{1} / P_{0}=0$, while the streamlines are quite different in these two cases.

If the pressure drop is small $\left(P_{1} / P_{0}=0.9\right)$ the gas forms a strong jet in the downflow container in the deep hydrodynamic regime $(\delta=100)$.

\section{CONCLUDING REMARKS}

The mass flow rate through a thin orifice for four values of the pressure ratio and in the whole range of the gas rarefaction was calculated by the direct simulation Monte Carlo method. The values of the pressure ration embrace the case of outflow into vacuum, i.e. a strong non-equilibrium flow, and the case of small pressure drop, i.e. weakly non-equilibrium flow.

It was shown that at any pressure ration the flow rate tends to a constant value in the hydrodynamic regime, which is established at the value of the rarefaction parameter $\delta$ equal to 20 . The most significant variation of the flow rate occurs in the range of the rarefaction parameter from 0.1 up to 20.

Near the free molecular regime and in the transition one the flowfield is almost symmetric for all values of the pressure ratio considered here. However, for large values of the rarefaction parameter, i.e. in the hydrodynamic regime, vortices appear in the downflow container if the pressure ratio differs from zero. At the small pressure drop, i.e. at $P_{1} / P_{0}=0.9$, the gas flow begins to form a strong jet by increasing the rarefaction parameter $\delta$.

To reach a reasonable numerical accuracy related to the statistical scattering the number of samples drastically increases by decreasing the pressure drop, i.e. when the pressure ration $P_{1} / P_{0}$ approaches to unity. In the range $0.9 \leq P_{1} / P_{0} \leq 1$ the CPU time increases so drastically that the DSMC method becomes inefficient.

It was observed a fine agreement between the present numerical results and experimental data at intermediate values of the pressure ratio.

\section{ACKNOWLEDGMENTS}

The research of the author is supported by the Conselho Nacional de Desenvolvimento Científico e Tecnológico (CNPq, Brazil).

\section{REFERENCES}

1. Sharipov, F., and Seleznev, V., J. Phys. Chem. Ref. Data, 27, 657-706 (1998).

2. Sharipov, F., Rarefied Gas Flow into Vacuum Through a Thin Orifice. Influence of the Boundary Conditions. accepted in AIAA J.

3. Sharipov, F., "Rarefied gas flow through a thin orifice," in Rarefied Gas Dynamics, edited by T. J. Bartel and M. A. Gallis, 22nd Int. Symp., Sydney, 2000, AIP, New York, 2001, pp. 494-501.

4. Bird, G. A., Molecular Gas Dynamics and the Direct Simulation of Gas Flows, Oxford University Press, Oxford, 1996.

5. Sreekanth, A. K., Phys. Fluids, 8, 1951-1956 (1965). 